\title{
Les Grands Aménagements Hydrauliques Transalpins
}

4 e Etude - 8e Article

\section{L'AMÉNAGEMENT GÉNÉRAL DE LA SCOLTENA}

\author{
Par J. BOUDET, Ingénieur A. \& M., Membre de la Société des Ingénieurs civils de France \\ et de $l$ "Associazione Elettrotecnica Italiana" (Sez. di Torino).
}

Nous n'entrerons pas dans le détail de ces calculs, dont l'exposé sortirait du cadre, forcément restreint, de cette étude d'ensemble. Ils nous paraissent suffisamment condensés dans les 2 épures ci-annexées (fig. 10 et 11 ), qui synthétisent la vérificalion de la stabilité de l'ouvrage : pour les arches (fig. 10) el pour les contreforts (fig. 11); par ailleurs, les caractéristiques les plus saillantes du travail constaté dans ces différentes parties dé l'ouvrage sont groupées ci-dessous (1):

Ainsi qu'on le verra dans le paragraphe suivant, l'ouvrage subit, au cours de sa construction, l'épreuve de terribles cléments naturels : crues subiles, tremblements de lerre, sans en ressentir le moindre dommage (2). Le seul rappel de ces circons-

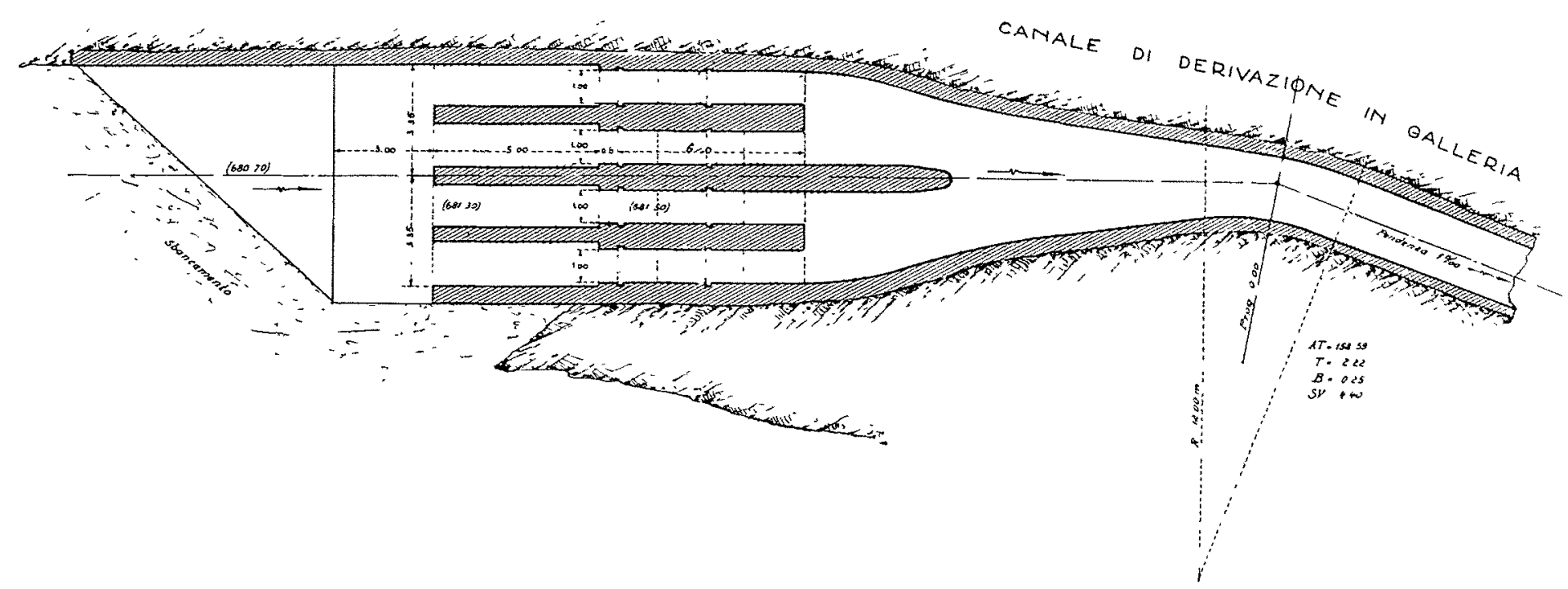

Fig. 8.- Prise d'eat: Coupe horizontale sur l'axe de la galerie d'amenée

\section{Distance du point de passage de la résultante d̀ celui de la \\ fibre moyenne sur \\ le plan de base du socle \\ Taux de travail maxima constates, au socle.

sur l'arête amont $\mid$ sur l'arête aval \\ I) Réservoir en charge normale $: \mathbf{R N}=687 \mathrm{~m}$. \\ $1 \mathrm{~m} .45$ (aval) | $3,5 \mathrm{kgs} / \mathrm{cmq}$ | I $8,3 \mathrm{kgs} / \mathrm{cmq}$. \\ II) Retenue exceptionnelle de crue : Alt. $688 \mathrm{~m} .50$.

$1 \mathrm{~m} .70$ (aval) | $3,2 \mathrm{kgs} / \mathrm{cmq}$. | $9,2 \mathrm{kgs} / \mathrm{cmq}$.

Efforts maxima $\quad \int$ à l'extrados : $15,2 \mathrm{kgs} / \mathrm{cmq}$. à la clef des voûtes $\left(^{*}\right) \quad$ à l'intrados : $18 \mathrm{kgs} / \mathrm{cmq}$.}

(*) Pour résister aux sollicitations à provenir des variations de température, les armatures transversales des arcs (aciers ronds)-sont doublées aux naissances des voûtes. tances nous paraît assez souligner la rationnalité de sa conception pratique autanl que de sa bonne exécution, qui font le plus grand honneur à notre éminenl collègue, l'ingénicur GanasSINI, auteur du projet el directeur des travaux de construction, ainsi qu'à l'Entreprise constructrice : Cottini-Palestro el $\mathrm{C}^{\text {ie }}$.

(1) Ceux de nos lecteurs qui désireraient suivre de plus près ce calcul - du reste fort intéressant - de barrage à arches multiples, consulteront, pensons-nous, avec fruit, notre étude sur la Rupture du Barrage du Gleno, publiée dans ces colonnes l'année dernière et dejà citée en renvoi ; de même que, à un point de vue beaucoup plus général, notre autre étude, actuellement en cours de publication, dans Les Nouvelles Annales de la Construction, sur "Une Nouvelle Contribution à l'Etude des Barrages en arc".

(2) Par ailleurs, en ce qui concerne les efforts inhérents tant aux variations de température qu'à la poussée des glaces, il résulte des communications personnelles; à nous faites par l'Ingénieur Ganassini, qu'ils sont absolument inoffensifs avec ce type de barrage : M. Ganassini a constaté, au Riolunato, plus de 15 degrés en-dessous de zéro et une épaisseur de glace dépassant $0 \mathrm{~m}$. 60, qui se rompait au fur et à mesure de sa formation -- par suite de la vidange du réservoir - déterminant autour des voûtes comme un " arc de rupture ", sans qu'il en résulte la moindre lésion, même capillaire dans les arches. 
d) Caractéristiques constructives; condilions el incidenls d'exécuiton. - Sans entrer dans de longs détails, nous rappellerons, ci-après, les conditions dans lesquelles ful exéculé le barrage de Riolunato el les incidents qui marquèrent son exécution :
Les contreforts sont en maconnerie ordinairc, avec parement en moellon smillé, au mortier de ciment à $300 \mathrm{kgs}$ et comportent, tous les 2 mètres de hauteur, sur $0 \mathrm{~m}$. 60 d'épaisseur $(2 \div 3$ assises maçonnées) fune lceinture en béton de ciment

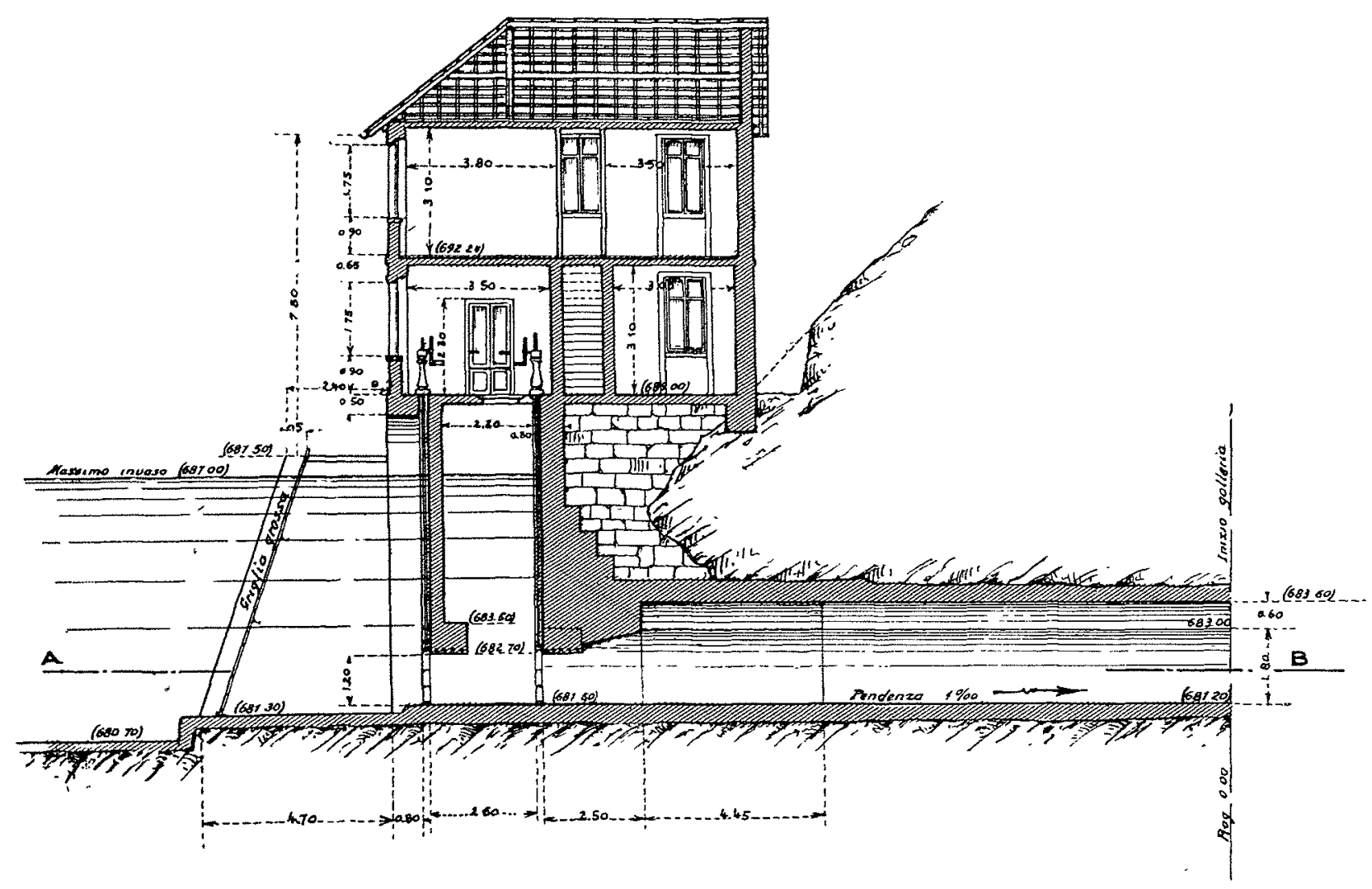

Fig. 9.- Prise d'ean : Coupe longitudinale sur l'axe de la galerie d'amenée

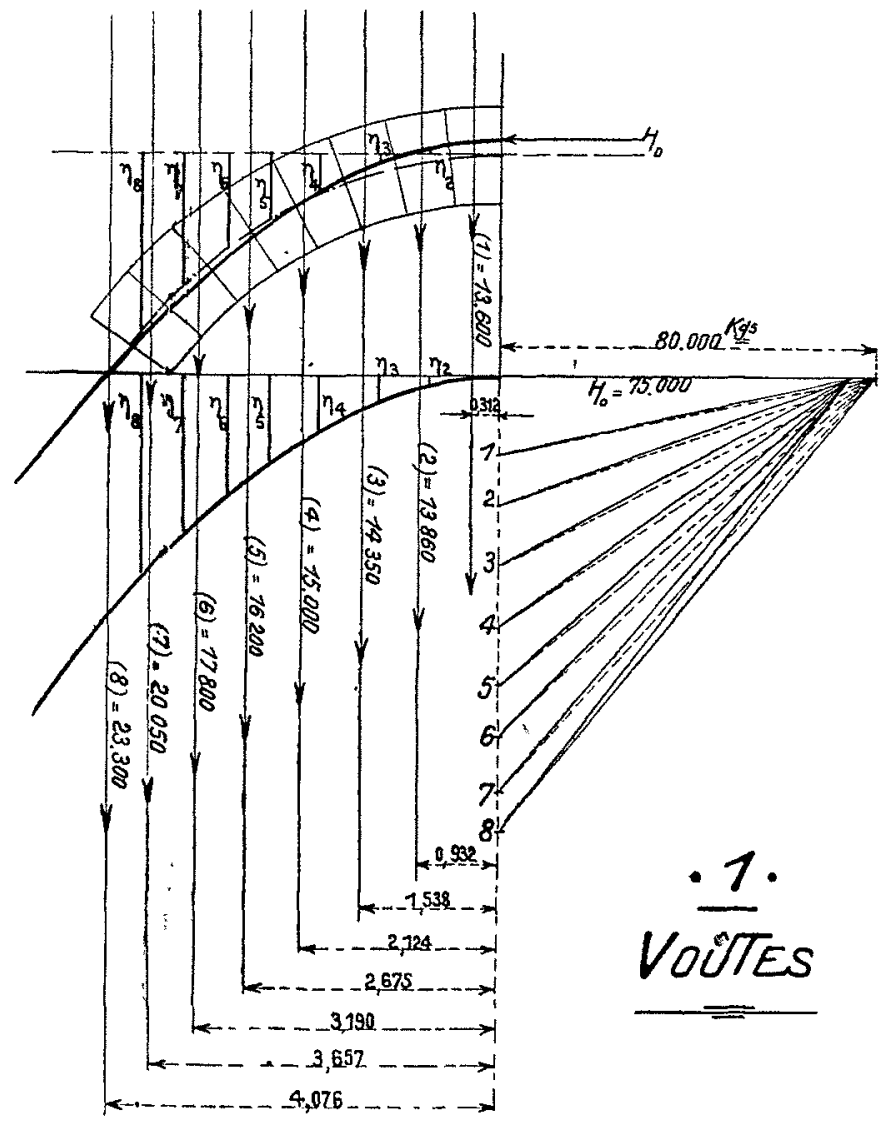

Fig. 10.- Vérification de la stabilité des voûtes

Matériaux : maconneries et béton. - Les massifs de fondation sont en beton de ciment à $250 \mathrm{kgs}$ de Portland par mètre cube de sable mouillé, à peinc sorti du lavage.

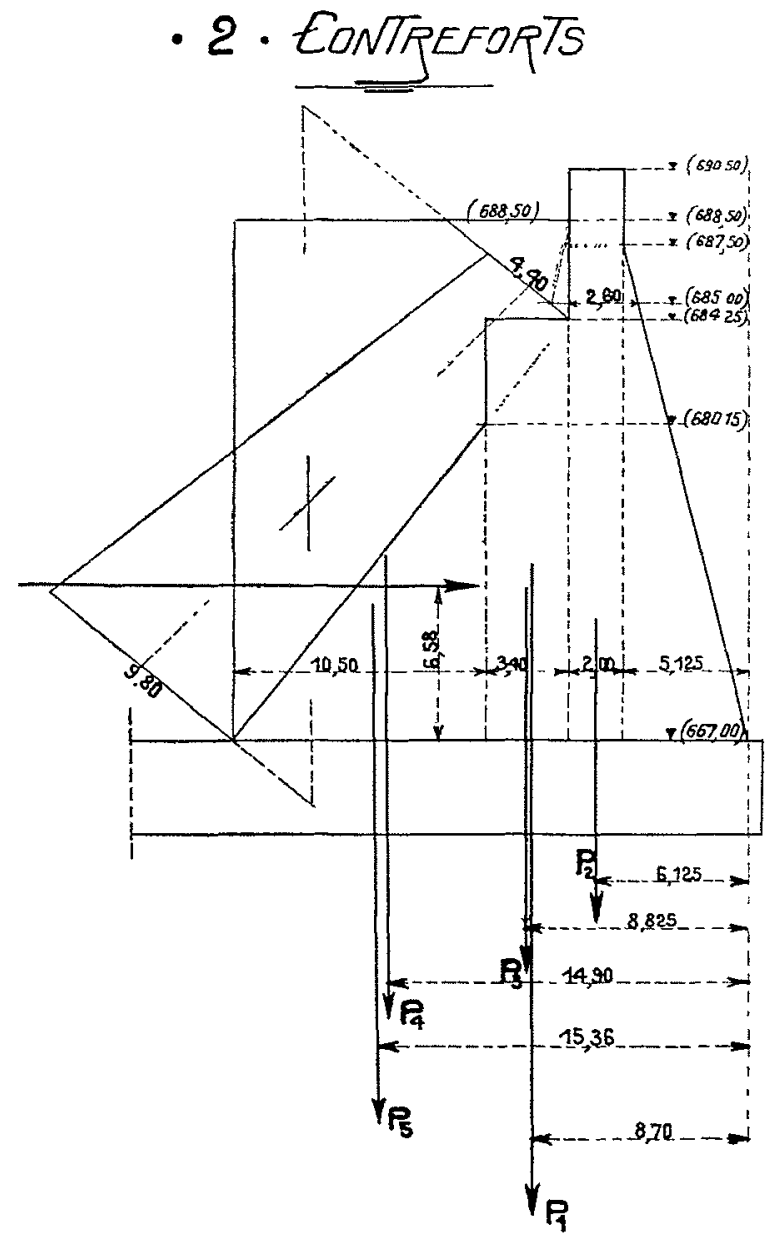

Fig. 11.- Vérification de la stabilité des contreforts

dosé à 250 kilos. Les appuis de rives (culées) sont en béton pour augmenter l'intimité de leur liaison avec le rocher. 
Les voûtes sont exclusivement en béton de ciment à forte teneur en sable, et dosé à $300 \mathrm{kgs.}$

Durée d'exćcution. - En dehors de l'économie importante qu'il permit de réaliser - (cubant seulement 10.000 mètres cubes (1), alors qu'un barrage du lype ordinaire à gravilé plein

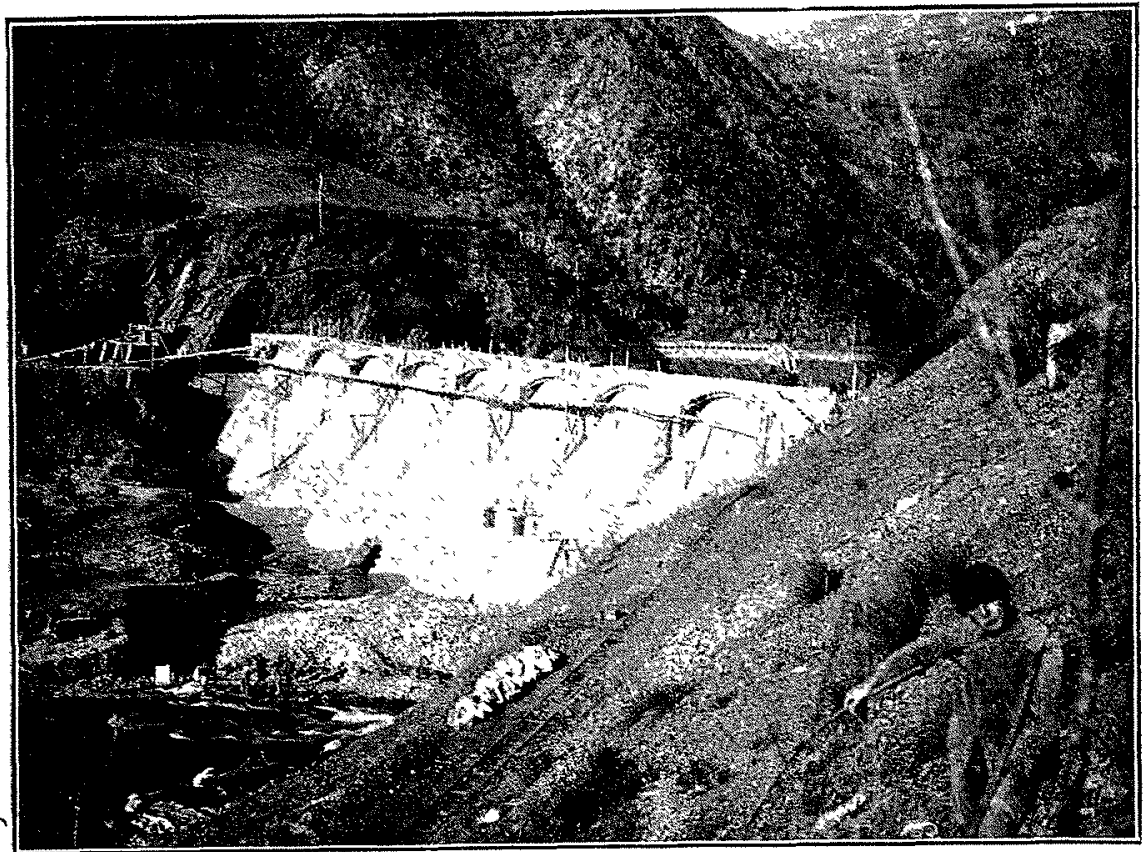

Photó 2.- Vue générale du Barrage du Riolunato prise, pendant la construction de l'ouvrage, à l'achèvement des voûtes.

eût cubé pour le moins $18.000 \mathrm{~m}^{3}$ ) - la caractéristique dominante de la construction de cet ouvrage réside dans la rapidité de son exécution qui, malgré les sérieux incidents que nous allons relater, put être virtuellement terminée dans 5 mois estivaux de travail effectif, grâce à une rationnelle organisation de chantiers.

Incidents d'exécution. - Les incidents qui marquèrent la construction du barrage, constituent, comme on va le voir, un criterium absolu de sa stabilité et de sa résislance. La plupart sont dûs à des circonstances atmosphériques, imprévisibles; elles firent courir de graves dangers à l'ouvrage, mais pour ne point entorser la vérité, nous devons ajouter que, pour certains, ces dangers furent accrus par diverses négligences et imprévisions ayant passé au travers de l'organisation des chantiers - pourtant bien comprise, dans son ensemble, par l'Entreprise constructrice... "Quandoque bonus dormitat Homerus $》$.

Parmi les plus importants, les premiers en date remontent au mois d'août 1920; ils sont dùs aux crues d'orages, aussi soudaines que terribles dans ces régions de hautes altiludes et qui, plusieurs fois, inondèrent les chantiers, remplissant de sable quelques fouilles el emportant différents boisages. A cette époque, les travaux de fondations étaient terminés et l'on commençait le bétonnage des voûles. Les travaux s'exécutaient à l'abri d'une dérivation du torrent, établie en rive droite, maís construile pour livrer

(1) Dont $1.400 \mathrm{~m}^{3}$ de béton de ciment pour les seules voutes. passage au senl débil caractéristique d'éliage; ce lul très insuflisant pour empecher leur submersion.

Par ailleurs, le bélonnage se poursuivail, pour parlie, sur des cintres en réemploi, que l'Entreprise utilisail, par mesure d'économie, pour n'avoir pas, à la fois, sur cintres, la totalité de l'ouvrage el i] faut bien reconnaître que son exécution traîna, alors qu'elle eûl dû être conduite rapidement jusqu’à achèvement complel. Ce fut une mauvase économie, qui en̂l pu conduire l'ouvrage à sa ruine lorsque, en septembre, alors qu'il étail presque achevé, et les chantiers en pleine activilé, une crue automnale, plus formidable encore que ses précédentes, lc surmonta, après l'avoir subitement mis en charge dans l'espace de quelques lieures.

A ce moment-là, - instruile par l'expérience des quẻlques crues qui avaient précédé celle-ci -- l'Entreprise, dans le but d'en faciliter l'écoulement éventuel, avail laissé, dans le pied de l'arche centrale (fond du thalweg) une fenêtre de 3 mètres de diamètre; en outre, les 2 pertuis des bondes de fond étaient demeurés ouverts. Tout cela -... joint à la galerie de dérivation - fut insuffisant, et la crue, montant rapidement jusqu'en crèle de l'ouvrage, se précipita par la brèche que constituait larche de rive gauche - en cours d'achèvement, - laquelle fil déversoir, livrant passage à une lame d'eau de 3 mètres d'épaisseur ! cependant que, par la fenêtre inférieure de l'arche centrale, des blocs de plus d'un mètre cube étaient roulés par la trombe, dans un fracas assourdissant, et projetés violemment sur la contre-digue du bassin-amortisseur, qui, élevée de fraîche date, ne put résister el s'effondra, laissant à l'eau sur les alluvions du fond du lit, une prise facilo

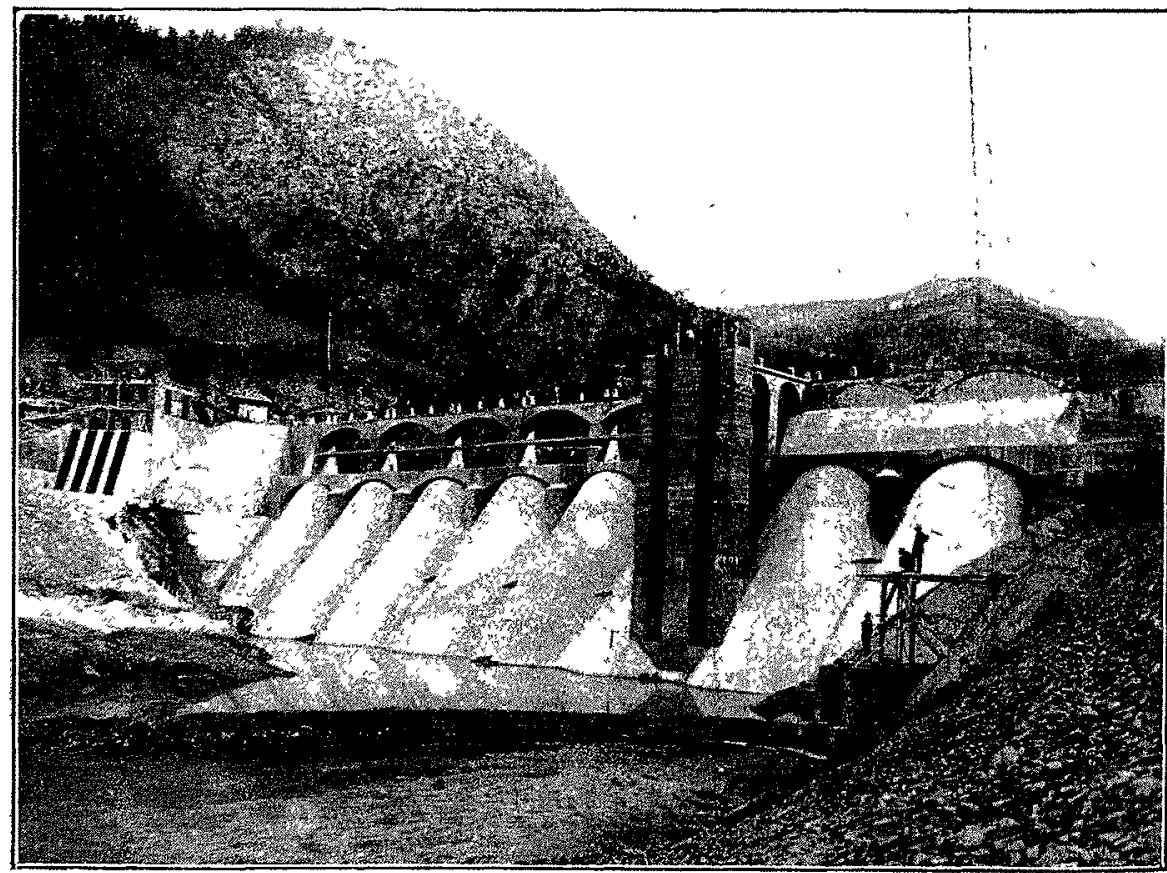

Photo 3.- Vue générale de l'ouvrage terminé (au premier plan, à la droite du lecteur, les bondes de fond et leur passerelle de manouvre; au fond, à sa gauche, la prise d'eau, surmontée par la maison du gardien). d'où découlèrent de sérieux affouillements pour les fondations des contreforts.

A cela, du reste, (abstraction faile du malériel el des bois emportés...) se bornèrent les effels de la crue sur l'ouvrage 
Iui-même qui, pendant prìs de 10 heures, supporta cetle terrible épreuve sans le moindre dommage pour ses cuvres vives. Mais le comble de la malchance ful dans une réitération de l'épreuve, survente en février 1921, du l'ail d'une crue printanière anticipéc qui, elle, dura plusieurs jours, alors que, par suile d'un manque prolongé de ciment sur le chanlier, on venail à peine d'oblurer la fenêtre ménagée au pied du barrage...

Lil galcrie, dans ces conditions, fonclionnait sous 18 mèlres de charge, el le canal d'évacuation, à la sortie du bassin-amortisseur, n'arrivail pas à lui fournir. Lorsque l'eau, submergeant la contre-digue, eul alteint un niveau supérieur au débouché aval de la galerie, il se produisil, dans la voûte de cette dernière partie où la paroi rocheuse présente l'épaisseur minimum une sorte d' "évent "qui, loul d'abord, donna quelques inquiéludes - heureusement injustifiées, - (1) pour la tenue du contre-

(1) Notre éminent collègue, l'Ingénieur Ganassini, assure qu'en cette circonstance - qui mit, pour quelques jours, ses nerfs à rude épreuve - il eut l'occasion rare d'avoir une idée vraiment nette de l'énorme résistance des voùtes : au cours de la démolition, seule la chape, de 3 centimètres d'épaisseur, était demeurée intacte dans une section d'environ 3 mètres de largeur et supportait, à elle seule, ainsi affaiblie, une pression de 4 mètres d'eau qui, par une ouverture pratiquée à la pince, projeta au dehors un jet rond et puissant parfaitement délimité. fort no 2, mais dont la conséquence la plus fâcheuse fut d'obliger à la réouverture de la fenêtre précédemment obturée, au pied du barrage, pour permettre son obturation en même temps que celle de la galerie.

Enfin, une fois réouverte la fenêtre de pred, on put monter une vanne au débouché de la galerie, oblurer ensuite la brêche, et, après quelques jours de séchage, meltre l'ouvrage en charge.

Depuis cette époque, il s'est toujours vaillamment comporlé, ne donnant prise à aucune critique de la part de l'Exploitation ; et il nous semble bien que son excellente tenue ne pourrait vraiment ètre mieux soulignée ici que par le rappel de l'épreuve terrible qui précéda sa réception : le tremblement de terre de Garfagnana, qui, dans la matinée du 7 septembre 1920, endeuilla, en quelques heures, toute la région émilienne, et qui, dans les environs immédiats des installations, détruisit presque complètement le petit pays de Riolunato, situé au bord du lac, provoquant sur ce dernier (le torrent étant en crue) des vagues de $2 \mathrm{~m}$. de hauteur, sans que la structure de l'ouvrage fut affectée par cet ébranlement formidable : l'examen -- des plus minutieux auquel fut soumis le barrage, aussitôt après, ne décela pas la moindre lésion, même capillaire !

\section{(A suivre)}

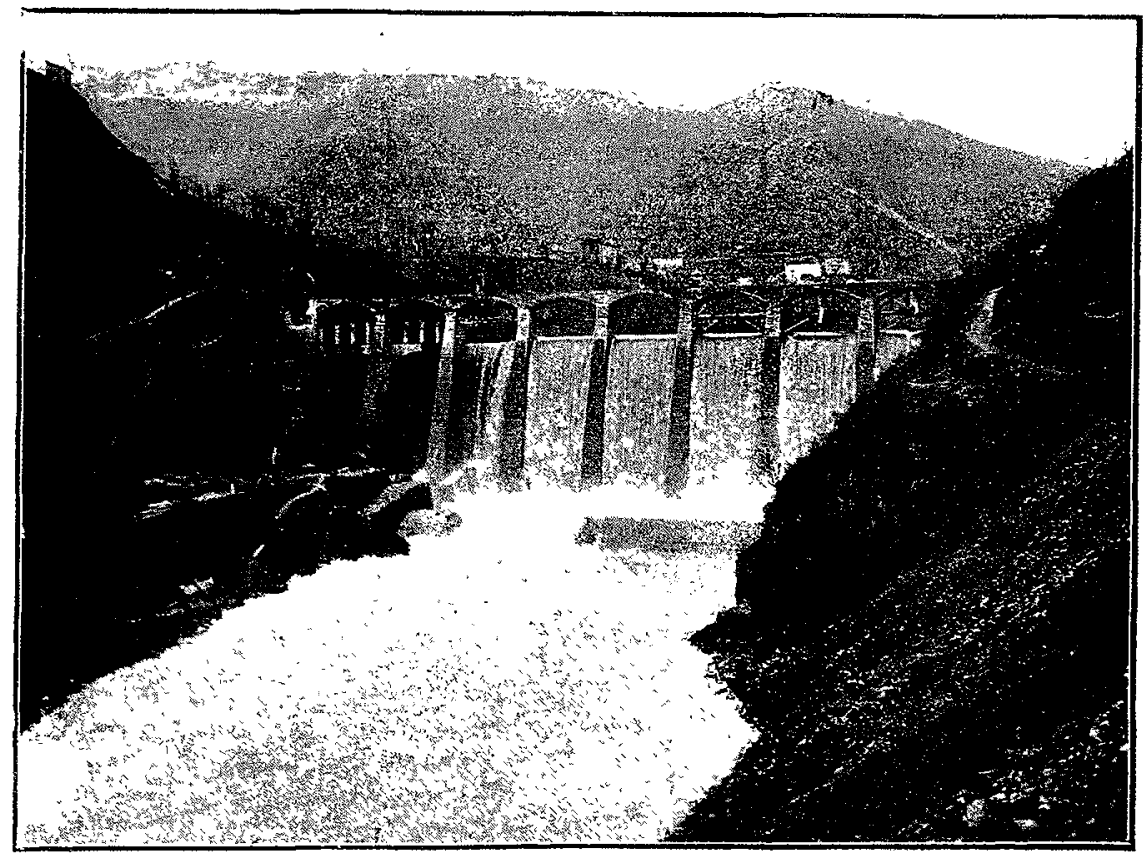

Photo 4. - Wue générale Septembre 1920, dont le déversement emporta une partie de la contredigue du bassin-amortisseur. 